\title{
Parenteral Nutrition Utilization in Bone Marrow Transplant Recipients
}

\author{
Wilson S*, Kohli-Seth R, Aldeguer Y, Pek M, Dharshan A, Oropello J, Manasia A, Bassily-Marcus A and \\ Benjamin E
}

Department of Surgery, Division of Surgical Critical Care Medicine, Icahn School of Medicine at Mount Sinai, New York, USA

*Corresponding author: Wilson S, Department of Surgery, Division of Surgical Critical Care Medicine, Icahn School of Medicine at Mount Sinai, One Gustave L. Levy Place, Box 1264, New York, NY 10029, USA E-mail: sara.wilson@mountsinai.org

Citation: Wilson S, Kohli-Seth R, Aldeguer Y, Pek M, Dharshan A, et al. (2014) Parenteral Nutrition Utilization in Bone Marrow Transplant Recipients. J Nutr Health Sci 1(1): 102. doi: 10.15744/2393-9060.1.102

Received Date: February 15, 2014 Accepted Date: May 15, 2014 Published Date: May 20, 2014

\begin{abstract}
Bone marrow transplant (BMT) recipients often require parenteral nutrition (PN) to meet their nutrient needs. While general guidelines for the provision of PN support by nutrition support teams (NSTs) have been shown to decrease inappropriate PN use, recommendations for nutrition in BMT recipients are lacking. We reviewed the charts of patients status post BMT on PN to determine whether institutional guidelines for PN initiation and continuous supervision of NSTs could be applied in this population. With the Institutional Review Board (IRB) approval, charts of adult BMT recipients on PN between June 14, 2006 and June 30, 2007 were examined. Sixty-nine charts were reviewed. Indications for initiation of PN included severe mucositis, graft versus host disease (GVHD), and other transplant related side effects resulting in poor oral intake. Among 69 patients, 37 (54\%) had severe mucositis, 12 (17\%) had GVHD, 2 (3\%) had both mucositis and GVHD, and 18 (26\%) had other side effects. It was determined that all patients met the criteria for initiation of PN support, as outlined in the guidelines form. Comprehensive guidelines for initiating PN support, developed by NSTs can also be used for BMT recipients in order to optimize their nutritional status.
\end{abstract}

Keywords: Total parenteral nutrition (TPN); Bone marrow transplant (BMT); Nutrition support; GVHD; Mucositis

\section{Introduction}

Parenteral nutrition (PN) is a specialized form of intravenous nutrition consisting of macro and micronutrients designed to meet the needs of patients who are unable to tolerate adequate enteral intake. When used appropriately PN can be a lifesaving therapy [1]. Bone marrow transplant is a well-established treatment modality for many diseases, including solid tumors, hematologic malignancies, and autoimmune disorders. Presently, there are two types of BMT that can be performed, autologous (a-BMT) and allogenic (allo-BMT) bone marrow transplantation. In patients who have undergone allo-BMT, 18-70\% develop acute graft versus host disease (GVHD) [2].This occurs when transplanted or grafted cells recognize the host as foreign, thereby initiating an immune response that causes diseases in the transplant recipient. Of those patients diagnosed with acute GVHD, 50\% progress to chronic GVHD, further increasing the risk for malnutrition and other related complications [3]. Intestinal GVHD characterized by diarrhea with or without nausea, vomiting, abdominal pain and occasionally ileus, contributes to the development of malnutrition, elucidating the need for PN use in these patients to meet their nutrient requirements [4].

Malnutrition is a negative prognostic factor for outcome after BMT. In patients undergoing BMT, impaired nutritional status can lead to longer engraftment time and greater probability of developing infection. Higher transplant-related mortality has also been observed in underweight patients (BMI <20) who undergo BMT $[5,6]$. The use of conditioning regimens has tremendous and deleterious consequences on the anatomical and functional integrity of the gastrointestinal tract. Likewise, the presence of infection and the drugs used for treatment and prophylaxis during the peri-transplant period can result in the development of mouth sores, nausea, vomiting and diarrhea. A common indication for PN use in BMT recipients is the occurrence of severe mucositis of the GI tract. It can affect up to 75\% of BMT recipients and combined with other gastrointestinal toxicities such as GVHD, and severe nausea and vomiting, it can significantly affect food intake and absorption resulting in dehydration and malnutrition [7].

The routine use of PN in BMT recipients, either as a supportive care or adjunctive therapy, minimizes the nutritional consequences of transplantation [8]. Despite overall favoring of enteral nutrition over PN, the presence of nausea, vomiting, and GI mucositis, make enteral nutrition support poorly tolerated by BMT patients. That coupled with the increased risk of bleeding associated with enteral tube placement in patients with thrombocytopenia, may justify the need for an alternative to enteral nutrition support. 
Providing prophylactic TPN during cytoreductive therapy to patients following transplantation has previously been shown to improve disease free and overall survival rates, as well as improve time to relapse [9]. Currently the data is limited and has not validated the benefits of either feeding route (PN vs EN) in these patients. Parenteral nutrition may be preferred in patients with severe GI complications that result in failed trials of enteral feeding [10]. Despite these practices, clear and established recommendations for when to appropriately initiate nutrition support are still lacking.

Guidelines from the European Society for Clinical Nutrition and Metabolism (ESPEN) published in 2009, report PN is ineffective in non-surgical oncology patients with a functional gastrointestinal tract, but recommend its use in patients with severe mucositis or severe radiation enteritis. For patients receiving hematopoietic stem cell transplant, PN should be reserved for patients with severe mucositis, ileus or intractable vomiting. Precise recommendations on the timing of initiation are unclear. They recommend discontinuing PN support when $50 \%$ of requirements are met enterally [11].

Guidelines for the Use of Parenteral and Enteral Nutrition published by The American Society for Parenteral and Enteral Nutrition (A.S.P.E.N.) in 2002 and The Society of Critical Care Medicine (SCCM) and A.S.P.E.N. Guidelines for the Provision and Assessment of Nutrition Support Therapy in the Adult Critically Ill published in 2009, also fail to provide specific recommendations for when BMT recipients should be provided with enteral and parenteral nutrition support. The 2002 A.S.P.E.N. guidelines acknowledged that peri-transplant patients often suffer transplant related side effects, which can alter their ability to meet nutrient needs enterally. The establishment of enteral access following ablative preparation regimens can be challenging. In patients who require PN support, A.S.P.E.N recommended transitioning to enteral nutrition as soon as side effects abate [12].

In May 2006, the Mount Sinai Hospital Medical Board initiated a quality assurance project whereby all parenteral nutrition consultations were reviewed for appropriateness. A PN review committee was formed and more than 600 charts were reviewed over the course of one year to evaluate whether a review committee coupled with the development of a guidelines form (Table 1) would lead to a decrease in the overall provision of inappropriate PN use in the hospital. With IRB approval, the charts of all adult patients who received PN from June 14, 2006 through June 30, 2007 were reviewed. Results showed that even with an already low rate, inappropriate PN use was further decreased by discussing inappropriate orders with the consulting team, educating staff and emphasizing use of the guidelines form. At that time, BMT patients were excluded from the review due to the lack of specific recommendations for the appropriate delivery of nutrition support in this population [13]. With the emergence of new literature that support appropriate PN use in BMT patients, these charts that were originally excluded were reviewed to determine whether PN administration would have been deemed appropriate using the criteria as outlined in the PN guidelines form.

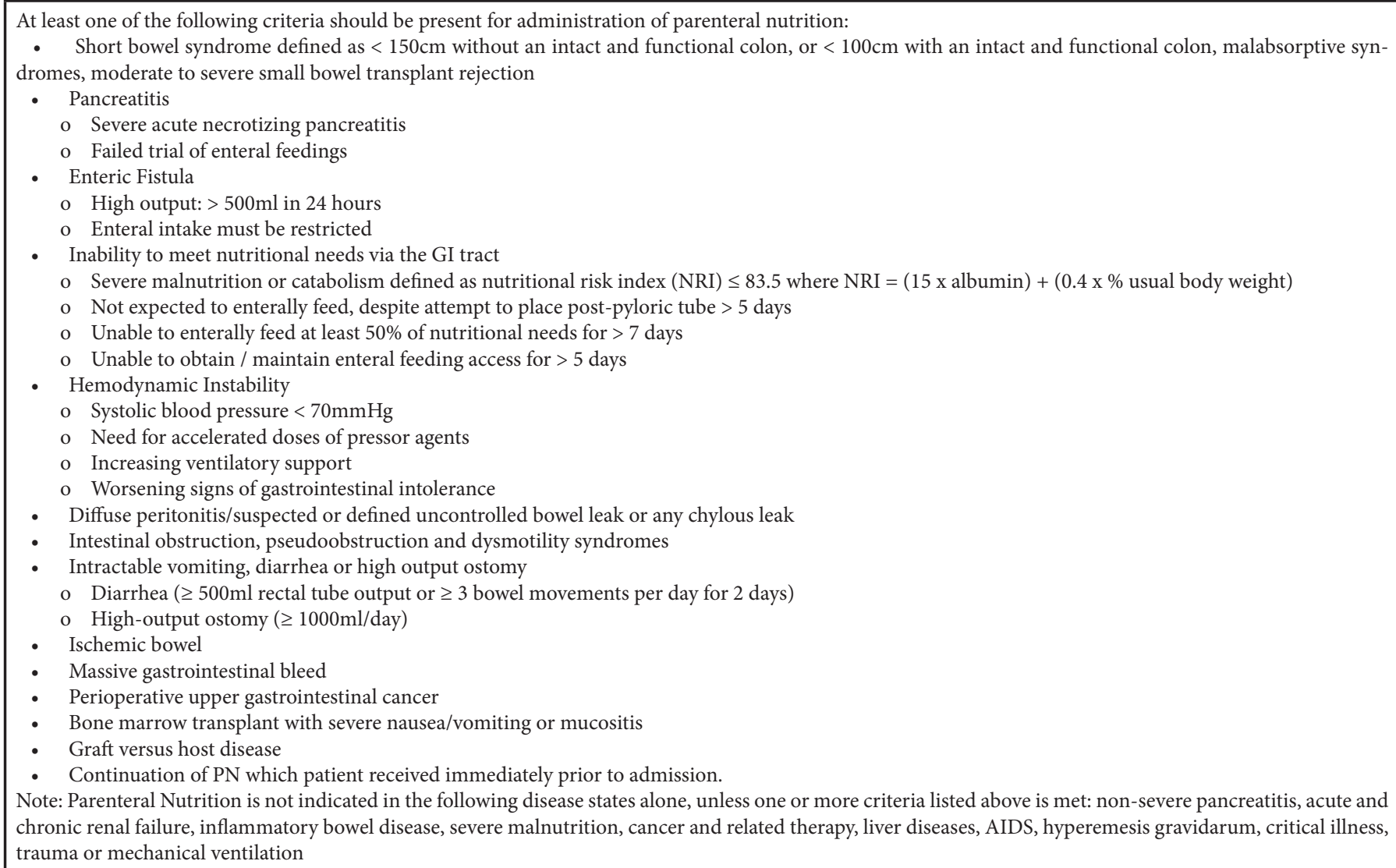

Table 1: Parenteral Nutrition Guidelines Form for Adults 


\section{Methods}

Mount Sinai Hospital is a tertiary care teaching hospital where PN is frequently administered. The majority of patients on PN are followed either by the surgical or medical nutrition support team (NST). Patients who undergo a BMT and require PN support are followed by an endocrinologist, who specializes in the provision of PN in this population, and a registered dietitian, who assesses the patient's nutritional status and ability to take in adequate calories and protein, enterally. The endocrinologist completes an assessment, determines whether PN is indicated, writes orders, and follows the patient for the duration of therapy.

With the IRB approval, we reviewed the 69 medical records of adult BMT recipients who received PN support between June 14, 2006 and June 30, 2007. Data on each patient was recorded by a registered dietitian and included, age, diagnosis, indication for PN, start date, duration of therapy and type of BMT. Duration of therapy was further classified as being short term, less than or equal to five days, or long term, greater than five days.

Our goal was to determine whether the same guidelines established by our original review committee could be applied to BMT patients despite a lack of definitive recommendations for providing PN support in this population.

\section{Results}

A total of 69 charts for patients status post BMT who received PN were reviewed. All patients were admitted by the Oncology service and placed on the BMT unit in the hospital. Thirty-seven patients were male (54\%). Mean patient age was 49 years (range 23-70). Thirty patients (43\%) underwent an autologous transplant and 39 (57\%) underwent an allogenic transplant. Twenty patients (29\%) were diagnosed with Non-Hodgkin's lymphoma (NHL), 13 (19\%) with multiple myeloma (MM), 12 (17\%) with acute myeloid leukemia (AML), 7 (10\%) with Hodgkin's lymphoma (HL), 6 (9\%) with acute lymphoblastic leukemia (ALL), 6 (9\%) with myelodysplastic syndromes, one with aplastic anemia, one with acute promyelocytic leukemia (APML), one with chronic lymphocytic leukemia (CLL), one with germ cell tumor, and one with desmoplastic small round cell tumor (Figure 1).

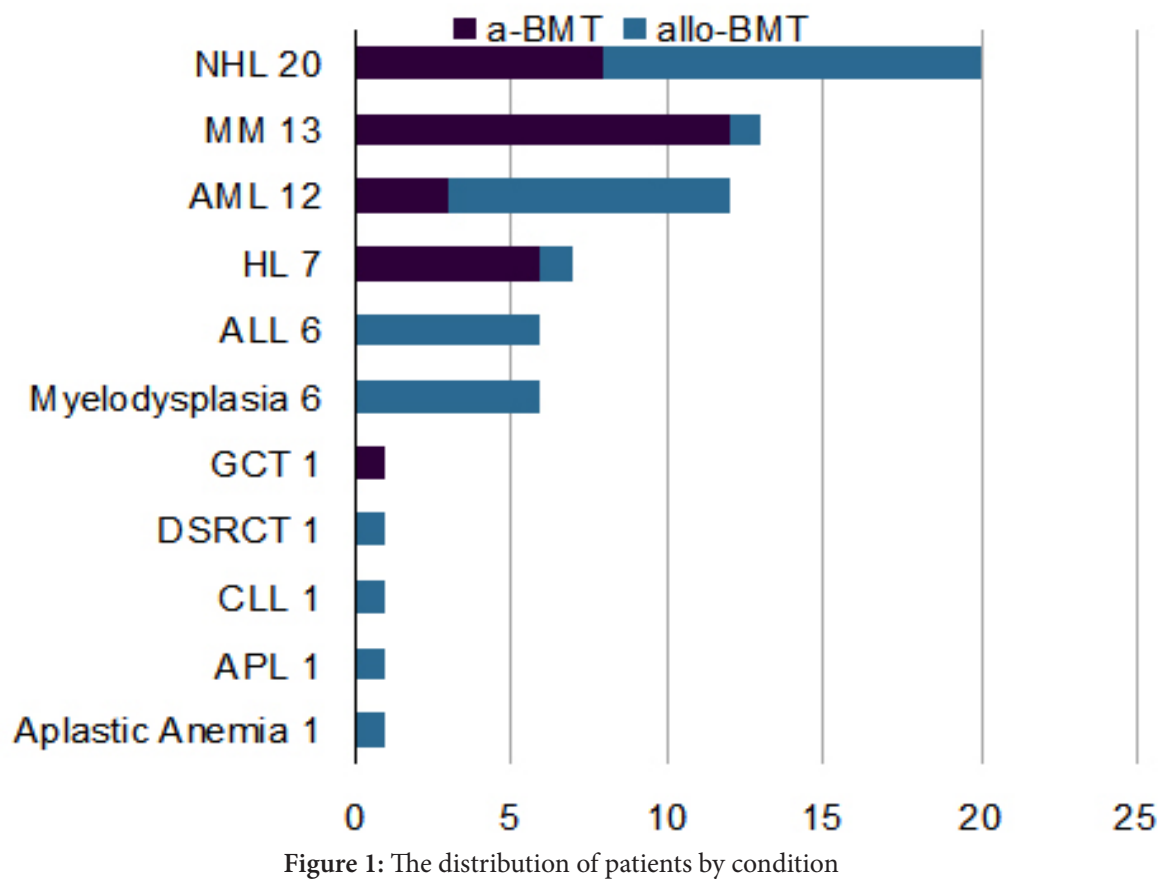

Indications for initiation of PN included severe mucositis, GVHD, and other transplant related side effects, including nausea, vomiting, and diarrhea that resulted in poor oral intake. Among the 69 patients, 37 (54\%) had documented severe mucositis with an inability to meet nutritional needs, 15 of which received an allogenic transplant and 22 an autologous transplant. Twelve patients (17\%) had documented GVHD and 2 (3\%) had both mucositis and GVHD. Of these 14 patients, all had received an allogenic transplant. The remaining 18 (26\%) had other transplant related side effects, which prevented the sufficient intake of nutrition. Among those patients 10 were allogenic transplant recipients and eight were autologous recipients. After reviewing the charts, $100 \%$ of PN starts were deemed appropriate based on the guidelines.

\section{Discussion}

A total of 105 patients underwent bone marrow transplantation in Mount Sinai Hospital between June 14, 2006 and June 30, 2007. During that same time period 69 patients were started on PN support for side effects following BMT that prevented them from taking sufficient calories to meet their nutrient requirements. 
After thorough review of patient charts, it was determined that all initiations of PN support met the criteria outlined in the guidelines form and newer guidelines set forth by ESPEN. This study confirms that our guidelines form was a comprehensive document that could be applied to all patient populations, including those where definitive guidelines have traditionally been lacking. These findings further support the development of criteria for PN initiation by NSTs. Involving multidisciplinary NST members that include ICU physicians, endocrinologists, registered dietitians, and pharmacists, integrates the principles of a diverse group of practitioners in order to develop standards that are not only useful as general guidelines, but also a valuable tool for a specialized group like BMT recipients, where explicit guidelines are lacking.

Complications related to PN were not assessed in this study. Many of our patients were critically ill and we found it difficult to definitively implicate $\mathrm{PN}$ as a cause for such complications as catheter-related sepsis and metabolic and electrolyte abnormalities in this group. Moreover, at our hospital, a central venous access team inserts all central catheters and monitors all mechanical and infectious complications related to their placement, and this team reported no complications during our study period. Our hospital also has a very low incidence of catheter-related sepsis and well-established glucose control protocols to maintain euglycemia [12].

In summary, BMT recipients routinely require PN support to meet their nutrient needs. While it is clear that multidisciplinary NSTs continue to play an important role in the management of PN support, written guidelines on when to initiate therapy in BMT patients are lacking. In a population where mortality is high, every effort should be made to optimize these patients. Establishing comprehensive guidelines developed by multidisciplinary NSTs that identify appropriate use of PN is critical.

\section{References}

1. Kitchen P, Forbes A (2003) Parenteral Nutrition. Curr Opin Gastroenterol 19: 144-7.

2. Iqbal N, Salzman D, Lazenby AJ, Wilcox CM (2000) Diagnosis of gastrointestinal graft-versus-host disease. Am J Gastroenterol 95: $3034-8$.

3. Jacobsohn DA, Vogelsang GB (2007) Acute graft versus host disease. Orphanet J Rare Dis 2: 35.

4. Cogbill CH, Drobyski WR, Komorowski RA (2011) Gastrointestinal pathology of autologous graft-versus-host disease following hematopoietic stem cell transplantation: a clinicopathological study of 17 cases. Mod Pathol 24: 117-25.

5. Rzepecki P, Barzal J, Sarosiek T, Szczylik C (2007) Biochemical indices for the assessment of nutritional status during hematopoietic stem cell transplantation: are they worth using? A single center experience. Bone Marrow Transplant 40: 567-72.

6. Le Blanc K, Ringden O, Remberger M (2003) A low body mass index is correlated with poor survival after allogeneic stem cell transplantation. Haematologica 88: 1044-52.

7. Vagliano L, Feraut C, Gobetto G, Trunfio A, Errico A, et al. (2011) Incidence and severity of oral mucositis in patients undergoing haematopoietic SCT--results of a multicentre study. Bone Marrow Transplant 46: 727-32.

8. Muscaritoli M, Grieco G, Capria S, Iori AP, Fanelli FR (2002) Nurtitional and metabolic support in patients undergoing bone marrow transplantation. Am J Clin Nutr 75: 183-90.

9. Weisdorf SA, Lysne J, Wind D, Haake RJ, Sharp HL, et al. (1987) Positive effect of prophylactic total parenteral nutrition on long-term outcome of bone marrow transplantation. Transplantation 43: 833-8.

10. Murray SM, Pindoria S (2009) Nutrition support for bone marrow transplant patients. Cochrane Database Syst Rev CD002920

11. Bozzetti F, Arends J, Lundholm K, Micklewright A, Zurcher G, et al. (2009) ESPEN Guidelines on Parenteral Nutrition: non-surgical oncology. Clin Nutr 28: 445-54.

12. A.S.P.E.N. Board of Directors and the Clinical Guidelines Task Force (2002) Guidelines for the use of parenteral and enteral nutrition in adult and pediatric patients. JPEN J Parenter Enteral Nutr 26: 1SA-138SA.

13. Kohli-Seth R, Sinha R, Wilson S, Bassily-Marcus A, Benjamin E (2009) Adult parenteral nutrition utilization at a tertiary care hospital. Nutr Clin Pract 24: 728-32. 